\title{
Case Report \\ Developing of Granulomatosis with Polyangiitis during Etanercept Therapy
}

\author{
María Clara Ortiz-Sierra, ${ }^{1}$ Andrés Felipe Echeverri, ${ }^{2}$ \\ Gabriel J. Tobón, ${ }^{2}$ and Carlos Alberto Cañas ${ }^{2}$ \\ ${ }^{1}$ Department of Internal Medicine, Fundación Valle del Lili, CES University Medicine School, Medellín, Colombia \\ ${ }^{2}$ Unit of Rheumatology, Fundación Valle del Lili, ICESI University Medicine School, Carrera 98 18-49, Cali, Colombia \\ Correspondence should be addressed to Carlos Alberto Cañas; cacd12@hotmail.com
}

Received 9 January 2014; Accepted 3 February 2014; Published 6 March 2014

Academic Editors: D. Melchiorre, C. Orellana, and C. Pineda

Copyright (C) 2014 María Clara Ortiz-Sierra et al. This is an open access article distributed under the Creative Commons Attribution License, which permits unrestricted use, distribution, and reproduction in any medium, provided the original work is properly cited.

\begin{abstract}
We describe a 67-year-old woman who developed c-ANCA positive vasculitis with involvement in eyes, skin, kidney, peripheral nerves, and upper and lower airway during treatment with etanercept therapy for rheumatoid arthritis. A diagnosis of Granulomatosis with Polyangiitis was done. Thus, anti-TNF therapy may be associated with the development of ANCA positive vasculitis.
\end{abstract}

\section{Introduction}

The tumor necrosis factor alpha (TNF- $\alpha$ ) is a proinflammatory cytokine involved in the pathogenesis of many inflammatory and autoimmune conditions as rheumatoid arthritis (RA) and inflammatory bowel disease among others. Several anti-TNF- $\alpha$ biological agents have been approved for the management of RA [1]. The most frequent adverse events related to anti-TNF- $\alpha$ therapies are infectious diseases, malignancies, demyelinating diseases, and drug-induced lupus [2, 3 ]. In addition, treatment with etanercept has been associated with the development of diverse granulomatous diseases such as sarcoidosis [4-6], granulomatous hepatitis [7], granulomatous thyroiditis [8], and Wegener's granulomatosis (now denoted as Granulomatosis with Polyangiitis-GPA-) [9]. Here, we report a new case of GPA developing in a patient with RA treated with etanercept.

\section{Case Report}

A 67-year-old woman was admitted in our service by a clinical picture of two weeks of new onset-very painful and erythematous nodules in lower limbs that were followed by ulceration. She additionally manifested constitutional symptoms, conjunctive injection, nasal congestion, paresthesia, and paresia in the lower limbs associated with peripheral neuropathy and polyarthritis. She had a 2-year history of RA refractory to treatment with prednisolone (10 mgr/day) and methotrexate ( $15 \mathrm{mgr} /$ weekly). After 8 months of this treatment and because of high activity disease score, a treatment with subcutaneous etanercept (50 mg weekly) was initiated three months before admission. The physical examination at admission revealed bilateral conjunctive injection, and the ophthalmologic exam showed bilateral scleritis. Deformity in hands with synovitis and nodules in the right elbow extensor aspect was also observed. Ulcerated lesions with necrotic center in lower limbs were evident (Figure 1). Laboratory test showed normal hemoglobin $(13 \mathrm{~g} / \mathrm{dL})$, high WBC, and platelets count $(18,000 / \mathrm{mL}$ and $542,000 / \mathrm{mL}$, resp.). No lymphopenia was found. Acute phase reactants were elevated, with an erythrosedimentation rate of $111 \mathrm{~mm} /$ hour and Creactive protein of $15.3 \mathrm{mg} / \mathrm{dL}$ (normal $<0.5 \mathrm{mg} / \mathrm{dL}$ ). Renal and liver functions were normal. The autoantibodies profile showed positivity for Rheumatoid Factor (45 IU/mL, cutoff $14 \mathrm{IU} / \mathrm{mL}$ ), antinuclear antibodies (1:320 homogeneous), and c-ANCA (1/320). The urinalysis showed hematuria without other relevant pathological findings. Multiple pulmonary parenchymal subpleural nodules were observed in chest radiography and CT (Figure 2). Additionally paranasal sinus CT was done showing chronic sinusopathy without 


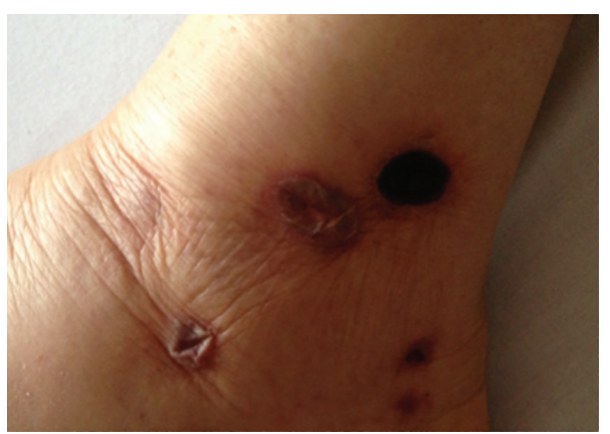

(a)

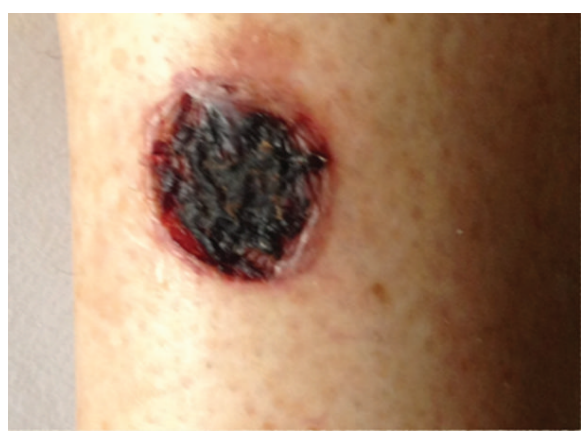

(b)

FIgURE 1: Vasculitic lesions with necrotic center in lower limbs in a patient with granulomatosis with polyangiitis.

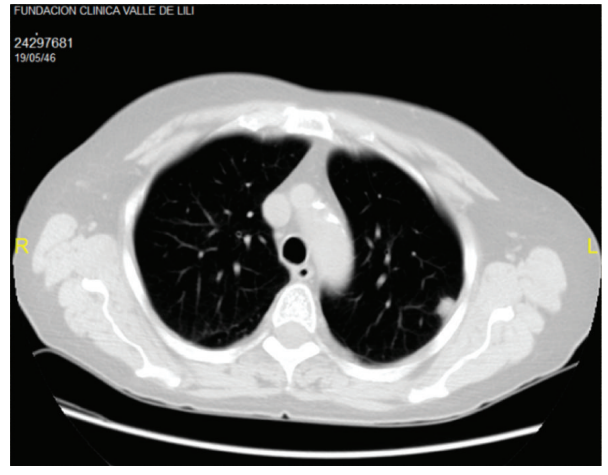

(a)

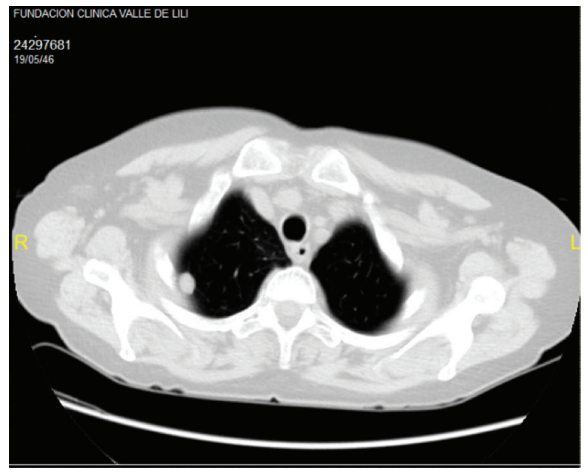

(b)

Figure 2: Pulmonary nodules seen on CT imaging of a patient with granulomatosis with polyangiitis.

bone erosions or cartilage destruction (Figure 3). A skin biopsy was performed showing inflammatory lesions with necrotic center consistent with leukocytoclastic vasculitis. A clinical GPA diagnosis was done. Treatment with methylprednisolone pulses and cyclophosphamide $750 \mathrm{mg}$ monthly was started, with subsequent steroid tapering regimen. At 3-month follow-up the patient presented a good clinical response with complete improvement of necrotic lesions, decrease in pulmonary nodules, and symptoms of upper airway.

\section{Discussion}

GPA (formerly named as Wegener's granulomatosis) is a systemic granulomatous disease associated with small and medium vessel vasculitis of unknown origin that primarily affects the upper respiratory tract, lungs, glomeruli, skin, kidneys, and peripheral nerves. The diagnosis is established based on clinical, radiological findings, presence of c-ANCA (by immunofluoresce), and anti-Proteinase 3 (PR3) antibodies by ELISA, supported by histological evidence of granulomas and vasculitis [10]. There is evidence that granuloma and vasculitis formation occurs in a genetically susceptible host as a result of autoimmune phenomena. Some experimental studies show that the c-ANCA (anti-PR3) antibodies are important to granuloma formation and chronic/refractory vascular involvement. Although the etiology of this disease is unknown, the most implicated environmental target is the bacterial infections, implicating $S$. aureus superantigens or Gram-negative bacteria [11]. In addition, some pharmacological molecules, including antithyroid drugs, may induce ANCA positivity. Also TNF- $\alpha$ is implicated in the GPA development. This cytokine plays a critical role in the immune system development and response regulation. Indeed, TNF- $\alpha$ stimulation primed the PR3 expression in mononuclear cells. This activation is needed to start the physiopathological axis in vasculitis.

Although different to the expected action of TNF- $\alpha$ in vasculitis, with the extended use of anti-TNF- $\alpha$ agents in diverse clinical scenarios, rare adverse events, including granulomatous diseases such as sarcoidosis and GPA, have been described. Thus, granulomatous diseases may be triggered or worsened by anti-TNF therapies $[8,9]$. Anti-TNF$\alpha$ may additionally trigger systemic autoimmune diseases as systemic lupus erythematosus [3].

Differences in the mode of neutralizing TNF- $\alpha$ action between these TNF- $\alpha$ antagonist may be implicated in the genesis of granulomatous reaction. Etanercept, a biologic agent composed of two recombinant forms of the human TNF receptor P75 fused to an Fc portion of human immunoglobulin G1, blocks primordially soluble TNF- $\alpha$ and has poor effect on tissue TNF- $\alpha$. The effect of Etanercept over 


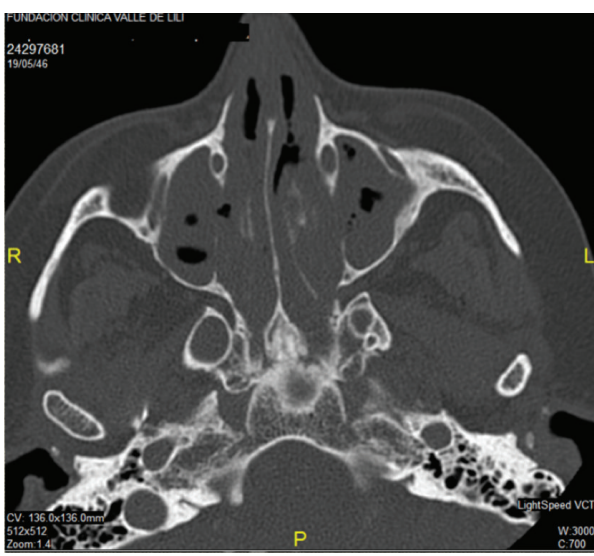

(a)

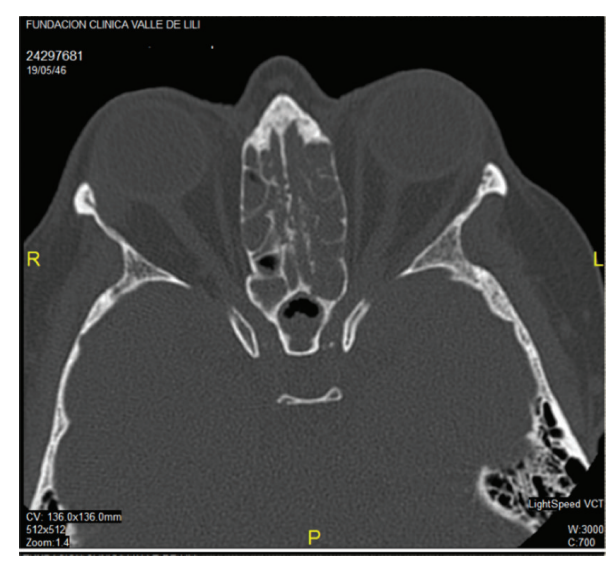

(b)

FIGURE 3: CT showing a diffuse sinus disease without erosions in a patient with granulomatosis with polyangiitis.

dilution of granulomas seems to be less important that those induce by antibodies against TNF- $\alpha$ such as infliximab that block this cytokine both tissue and soluble forms [12].

Although less expected as an adverse event, GPA development has been described with anti-TNF- $\alpha$ therapies such as etanercept [9] and golimumab [13]. However, the underlying mechanism of granulomatous conditions after anti-TNF- $\alpha$ exposure is not fully explained. Cytokine misbalance after TNF- $\alpha$ inhibition may induce granuloma formation in a compensatory way $[4,14]$.

In conclusion, we reported a new case of GPA developing during etanercept therapy. This clinical observation must be considered in all patients treated with anti-TNF.

\section{Conflict of Interests}

The authors declare that there is no conflict of interests regarding the publication of this paper.

\section{References}

[1] F. Conti, F. Ceccarelli, L. Massaro et al., "Biological therapies in rheumatic diseases," Clinical Therapeutics, vol. 164, no. 5, pp. e413-e428, 2013.

[2] M. C. Hochberg, M. G. Lebwohl, S. E. Plevy, K. F. Hobbs, and D. E. Yocum, "The benefit/risk profile of TNF-blocking agents: findings of a consensus panel," Seminars in Arthritis and Rheumatism, vol. 34, no. 6, pp. 819-836, 2005.

[3] J. C. Diaz, S. Vallejo, and C. A. Cañas, "Drug-induced lupus in anti-TNF-alpha therapy and its treatment with rituximab," Rheumatology International, vol. 32, no. 10, pp. 3315-3317, 2012.

[4] T. Ishiguro, N. Takayanagi, K. Kurashima et al., "Development of sarcoidosis during etanercept therapy," Internal Medicine, vol. 47, no. 11, pp. 1021-1025, 2008.

[5] K. E. Hostettler, U. Studler, M. Tamm, and M. H. Brutsche, "Long-term treatment with infliximab in patients with sarcoidosis," Respiration, vol. 83, no. 3, pp. 218-224, 2012.
[6] A. Kudrin, E. R. Chilvers, A. Ginawi et al., "Sarcoid-like granulomatous disease following etanercept treatment for rheumatoid arthritis," Journal of Rheumatology, vol. 34, no. 3, pp. 648649, 2007.

[7] M. Farah, A. Al Rashidi, D. A. Owen, E. M. Yoshida, and G. D. Reid, "Granulomatous hepatitis associated with etanercept therapy," Journal of Rheumatology, vol. 35, no. 2, pp. 349-351, 2008.

[8] C. A. Cañas, G. J. Tobón, L. G. Arango, and N. Guarín, "Developing of granulomatous thyroiditis during etanercept therapy," Clinical Rheumatology, vol. 28, supplement 1, pp. S17S19, 2009.

[9] V. Broshtilova, E. Iliev, and M. Gantcheva, "Etanercept-induced Wegener granulomatosis in a patient with rheumatoid arthritis," Dermatologic Therapy, vol. 26, pp. 73-76, 2013.

[10] R. Y. Leavitt, A. S. Fauci, D. A. Bloch et al., "The American College of Rheumatology 1990 criteria for the classification of Wegener's granulomatosis," Arthritis and Rheumatism, vol. 33, no. 8, pp. 1101-1107, 1990.

[11] H. Tadema, P. Heeringa, and C. G. M. Kallenberg, "Bacterial infections in Wegener's granulomatosis: mechanisms potentially involved in autoimmune pathogenesis," Current Opinion in Rheumatology, vol. 23, no. 4, pp. 366-371, 2011.

[12] B. Scallon, A. Cai, N. Solowski et al., "Binding and functional comparisons of two types of tumor necrosis factor antagonists," Journal of Pharmacology and Experimental Therapeutics, vol. 301, no. 2, pp. 418-426, 2002.

[13] K. Parekh, D. Ching, M. U. Rahman, and L. K. Stamp, "Onset of Wegener's granulomatosis during therapy with golimumab for rheumatoid arthritis: a rare adverse event?" Rheumatology, vol. 49, no. 9, pp. 1785-1787, 2010.

[14] A. Massara, L. Cavazzini, R. La Corte, and F. Trotta, "Sarcoidosis appearing during anti-tumor necrosis factor alpha therapy: a new "class effect" paradoxical phenomenon. Two case reports and literature review," Seminars in Arthritis and Rheumatism, vol. 39, no. 4, pp. 313-319, 2010. 


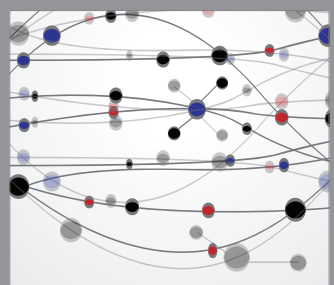

The Scientific World Journal
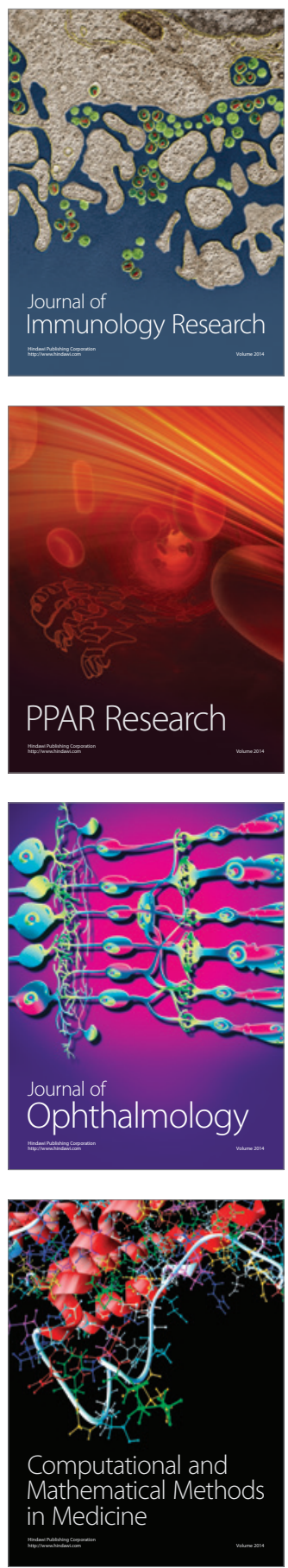

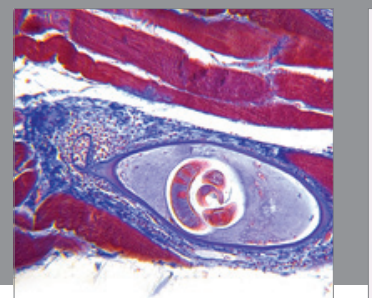

Gastroenterology

Research and Practice
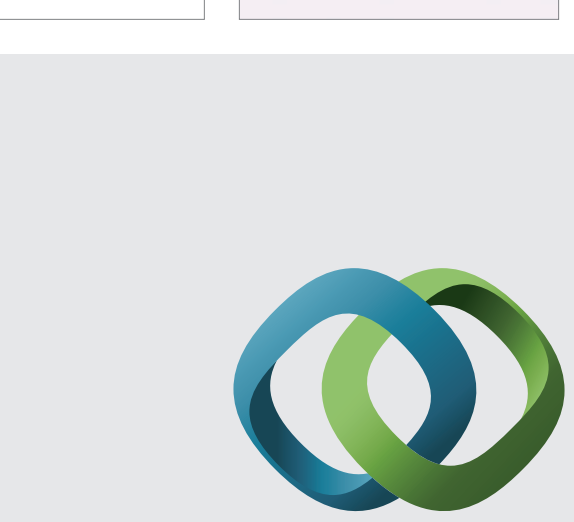

\section{Hindawi}

Submit your manuscripts at

http://www.hindawi.com
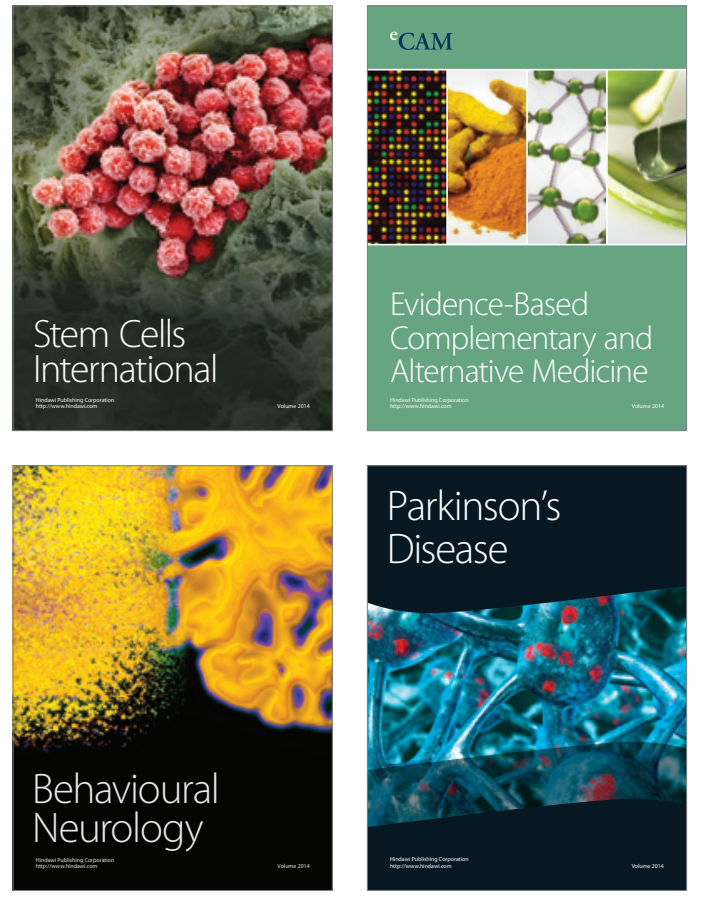
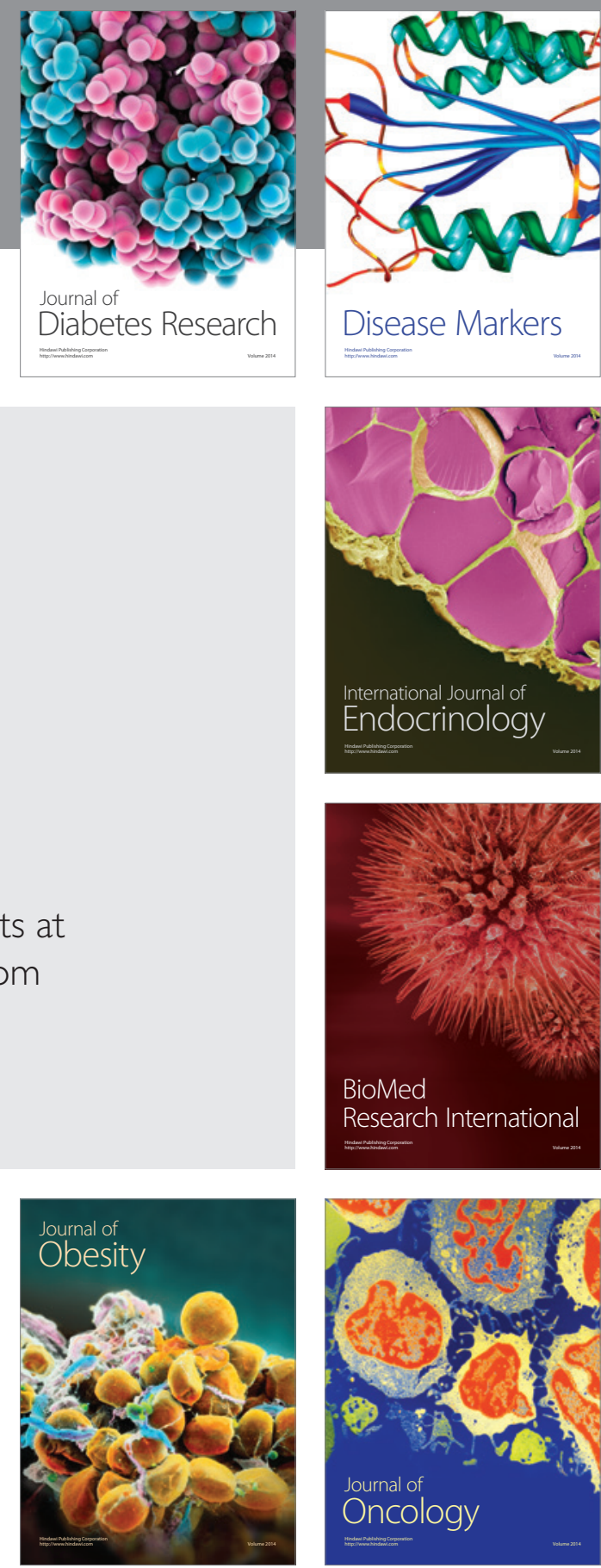

Disease Markers
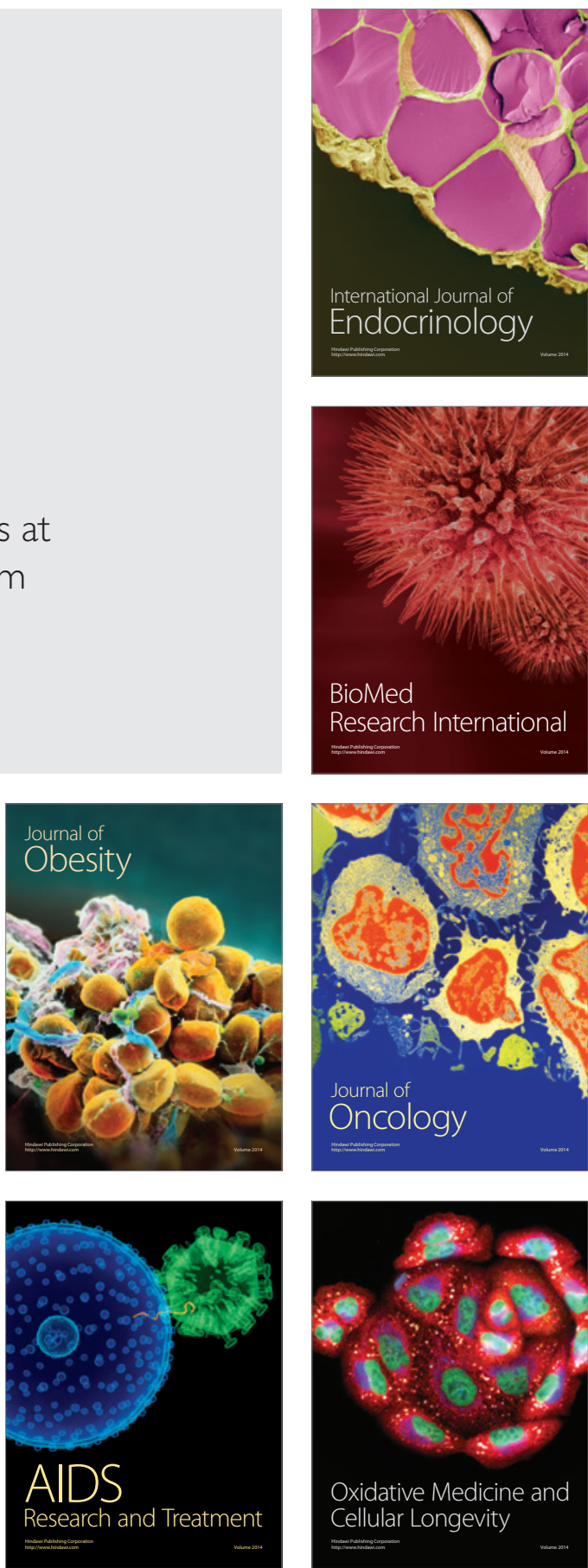